Document downloaded from:

http://hdl.handle.net/10251/81841

This paper must be cited as:

Camba, JD.; Contero, M.; Salvador Herranz, GM.; Plumed, R. (2016). Synchronous communication in PLM environments using annotated CAD models. Journal of Systems Science and Systems Engineering. 25(2):142-158. doi:10.1007/s11518-016-5305-5.

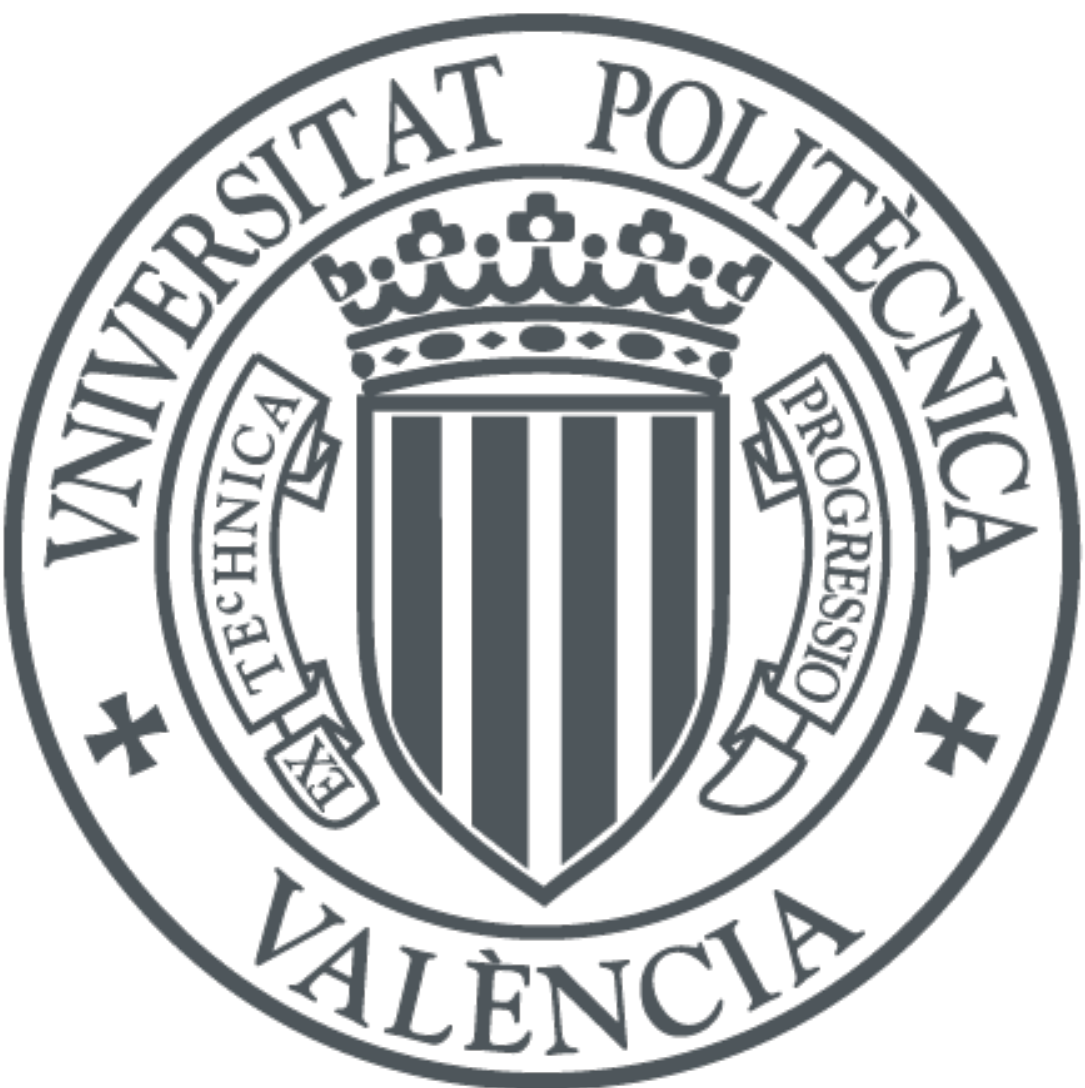

The final publication is available at

http://doi.org/10.1007/s11518-016-5305-5

Copyright Springer Verlag (Germany)

Additional Information 
Camba, J. D., Contero, M., Salvador-Herranz, G., \& Plumed, R. Synchronous communication in PLM environments using annotated CAD models. Journal of Systems Science and Systems Engineering, 1-17.

DOI:10.1007/s11518-016-5305-5

The final publication is available at springerlink.com:

http://link.springer.com/article/10.1007\%2Fs11518-016-5305-5 


\title{
Synchronous Communication in PLM Environments using Annotated CAD Models
}

\author{
Jorge D. Camba ${ }^{1} \quad$ Manuel Contero $^{2} \quad$ Gustavo Salvador-Herranz ${ }^{3}$ Raquel Plumed $^{4}$ \\ ${ }^{1}$ Dwight Look College of Engineering, Texas A\&M University, College Station, TX \\ camba@tamu.edu $(\square)$ \\ ${ }^{2}$ I3BH, Universitat Politècnica de València, Valencia, Spain \\ mcontero@upv.es \\ ${ }^{3}$ Depto. de Expresión Gráfica, Proyectos y Urbanismo, Universidad CEU Cardenal Herrera, Valencia, Spain \\ gsalva@uch.ceu.es \\ ${ }^{4}$ Depto. de Ingeniería Mecánica y Construcción, Universitat Jaume I, Castellón, Spain \\ plumed@uji.es
}

\begin{abstract}
The connection of resources, data, and knowledge through communication technology plays a vital role in current collaborative design methodologies and Product Lifecycle Management (PLM) systems, as these elements act as channels for information and meaning. Despite significant advances in the area of PLM, most communication tools are used as separate services that are disconnected from existing development environments. Consequently, during a communication session, the specific elements being discussed are usually not linked to the context of the discussion, which may result in important information getting lost or becoming difficult to access. In this paper, we present a method to add synchronous communication functionality to a PLM system based on annotated information embedded in the CAD model. This approach provides users a communication channel that is built directly into the CAD interface and is valuable when individuals need to be contacted regarding the annotated aspects of a CAD model. We present the architecture of a new system and its integration with existing PLM systems, and describe the implementation details of an annotation-based video conferencing module for a commercial CAD application.
\end{abstract}

Keywords-Collaborative engineering; PLM; design communication; CAD integration; video conferencing.

\section{INTRODUCTION}

In today's globalized and knowledge-based economies, engineering organizations rely as much on their collective body of knowledge and experience as their physical and economic assets (Ping-Hung et al., 2003). Engineering design is a complex and multidisciplinary activity that depends largely on a skilled workforce possessing "know how," "know what," and "know why." Like other types of knowledge work, specialists from various disciplines must work together to accomplish specific goals, increase performance, and reduce development time and costs without compromising quality. Success in engineering design not only requires that technical needs be met, but they must also be met efficiently and effectively (Alavi and Tiwana, 2002).

The constant pressure put on organizations and ever increasing customer's expectations for rapidly delivering high-quality products at competitive prices require new methods, tools, and a more efficient use of resources and technology to meet the demands and gain or maintain competitive advantages (Toussaint and Cheng, 2002). For this reason, modern engineering teams are gradually replacing traditional centralized product development paradigms with more efficient concurrent methodologies, where teams share knowledge and work collaboratively in virtual environments. This shift in methodologies has allowed a better integration of design, manufacturing, and other related tasks as well as a reduction of development times by maximizing the parallelization of working practices (Levitt et al., 1991). 
Communication is a critical element in collaborative engineering activities for connecting ideas and developing a shared understanding of the final design product, as a considerable portion of engineers' time is spent sharing and exchanging information (Lahti et al., 2004; Gupta et al., 2009). In this context, the integration of resources, data, and knowledge through communication is vital, as these elements act as channels for information and meaning, and have a direct impact in many engineering outcomes (Danesi et at., 2006; Barley et al., 2012). As engineering projects become larger, more distributed, and increasingly more complex, the role of specialized tools to support design activities becomes more relevant (Chudoba et al., 2005; Fuh and Li, 2005). Consequently, organizations continue to make significant investments in information technology specifically designed to support the sharing of knowledge (Wasko and Faraj, 2005). These technologies range from simple document repositories to comprehensive knowledge management applications, Product Lifecycle Management (PLM) systems, and advanced Information and Computer Technologies (ICT) (Chudoba et al., 2005; Fuh and Li, 2005).

In recent years, many authors have proposed different uses of computer technology to support both synchronous and asynchronous communication in virtual design environments (Tsoi and Rahman, 1996; Cutkosky et al., 1996; Durstewitz et al., 2002; Fonseca et al., 2006). A number of experimental design spaces such as Virtual Design Studio (Tay and Ming, 2001) and CyberCAD (Tay and Roy, 2003), and other integrated tools for collaborative CAD have also been developed. Commercial CAD packages, however, are still dominant players in professional production environments. In a PLM setting, communication can be managed by Computer Supported Collaborative Work (CSCW) modules, one of the major functional blocks of the PLM system. Some of the functionality provided by these modules includes messaging, videoconferencing, and electronic blackboards.

In this paper, we review the use of communication technology, particularly video conferencing, as a tool to support information exchange in collaborative engineering environments. We examine user-to-user communication and discuss the lack of integration of communication tools with actual design applications. We argue that this disconnection makes it difficult to address specific challenges and design tasks in a purely collaborative manner. We justify the importance of a full integration and propose a model to incorporate CSCW technology within existing Computer-Aided Design (CAD) packages so that all communication activities are funneled through annotated CAD models. Finally, the architecture of a software component that provides real time communication (messaging and video/audio) capabilities to CAD systems is described. The component interacts directly with the CAD package and can be added to existing PLM systems.

\section{RELEVANT Literature}

\section{A. Communication in Product Lifecycle Management (PLM) Systems}

The size and complexity of today's pressing design challenges requires collaborative efforts in which a successful combination of individual solutions must be achieved. As product development projects become more demanding, the idea of a solitary engineer able to master all the intricacies of the project is no longer viable (Arias et al., 2000). Instead, teams of designers, even when geographically dispersed (You and Chao, 2006), must work simultaneously on a variety of tasks while using the most up-to-date documents and drawings (Abrahamson et al., 2000; Vila et al., 2007). Therefore, capturing, processing, and managing all the information that is exchanged during collaborative activities, as well as making this information easily accessible to the appropriate teams, are necessary prerequisites for success (Ahmed, 2005) and may speed up future design projects by enabling better design reuse (Bai et al., 2010). In this regard, Product Lifecycle Management (PLM) systems play a fundamental role in modern product development and engineering design processes.

Distributed design environments have been transformed from single standalone workstations to a combination of software systems, services, and infrastructures ( $\mathrm{Li}$ et al., 2005) that support collaboration, communication, and content management. Specifically, collaboration is supported by increasing responsiveness, information availability, and mass customization; communication is facilitated by reducing transfer times and data navigation iterations; and content management is improved by providing consistent and accurate product definition and helpful product viewing (Toussaint and Cheng, 2002). According to (Fonseca et al., 2006), software applications involved in collaborative design can be categorized in three groups:

- Functional Applications, such as CAD/CAM packages, used for content creation and development of any product or process.

- Management Applications, such as PLM systems, used to administer, control, support, and manage the data created by the functional applications. 
- Communication Applications, such as email, messaging services, or videoconferencing, used to support communication among teams.

Product Lifecycle Management (PLM) refers to the activity of managing, in the most effective way, all product related information and processes across the enterprise throughout its entire lifecycle, from initial concepts to retirement and disposal (Stark, 2011). It is a concept that integrates data, users, processes, and systems, providing a product information framework for the extended enterprise. As a technology solution, it establishes a set of tools and technologies that provide a shared platform for collaboration among product stakeholders and streamlines the flow of information along all the stages of the product life cycle (Ameri and Dutta, 2005).

The diverse nature of PLM systems has promoted the development of new collaborative design tools, which combine CAD, Product Data Management (PDM), and Web access in an integrated environment. According to (Sharma, 2005), collaboration at the highest level could be viewed as a framework to connect People, Processes and Information. This connection cannot exist without an underlying PLM structure that facilitates communication, as it is through communication that teams make sense of the information needed for their work, their shared sense of who knows what, and the shared meaning of the ideas necessary to knowledge work (Yuan et al., 2010). Therefore, a robust communication infrastructure, typically based on Internet technology, is a necessary condition for the success of a distributed collaborative environment (Londono et al., 1992; Upton and Mcafee, 1999).

From a communication technology standpoint, creative dialogs and technical discussions can be established by PLM users in a variety of forms, including chats, forums, instant messages, videoconferencing, or screen sharing sessions (Leenders et al., 2003). Naturally, all these services are readily available in the PLM environment, which offers a number of tools that facilitate information exchange and virtual meetings among designers, often in the form of web-based applications (Chudoba et al., 2005; Tavčar et al., 2013). In most cases, however, the tools are separate from the design system and shared CAD models, and frequently run as standalone desktop applications or web services. Examples include email clients, instant messaging applications, and telecommunications software. As a result, the specific elements being discussed are usually not linked to the context of the discussion, which may result in important information becoming difficult to access or getting lost in email inboxes or voice mail recordings (Siltanen and Valli, 2013). In fact, despite engineering firms' significant investments in PLM technology, email remains one of the most popular and widely used information sharing tools (Gantz et al., 2007) and has become central to working processes in enterprises (Fisher et al., 2006; Wasiak et al., 2010). Despite its asynchronous nature and intrinsic limitations to efficiently manage large volumes of information, many organizations are acknowledging the value of email exchanges as an information source and are starting to offer support and develop tools to take advantage of this resource (Wasiak et al., 2010).

In an industrial setting, a PLM system is typically a multiplatform and multivendor environment, which means that communication standards are critical to ensure interoperability. In this regard, a number of standards have been developed by the International Telecommunication Union and the International Multimedia Teleconferencing Consortium. The standards for multimedia conferencing include recommendations related to both the communication and collaboration layers, as summarized by (Contero et al., 2002) and shown in Figure 1.

Recommendations related to data conferencing include T.126, the multipoint still image and annotation protocol; T.127, the multipoint binary file transfer protocol; T.128, for multipoint application sharing; and T.134, a text chat application entity. In terms of audio and videoconferencing, recommendations include standards for communication bandwidth, video codec, and call control 

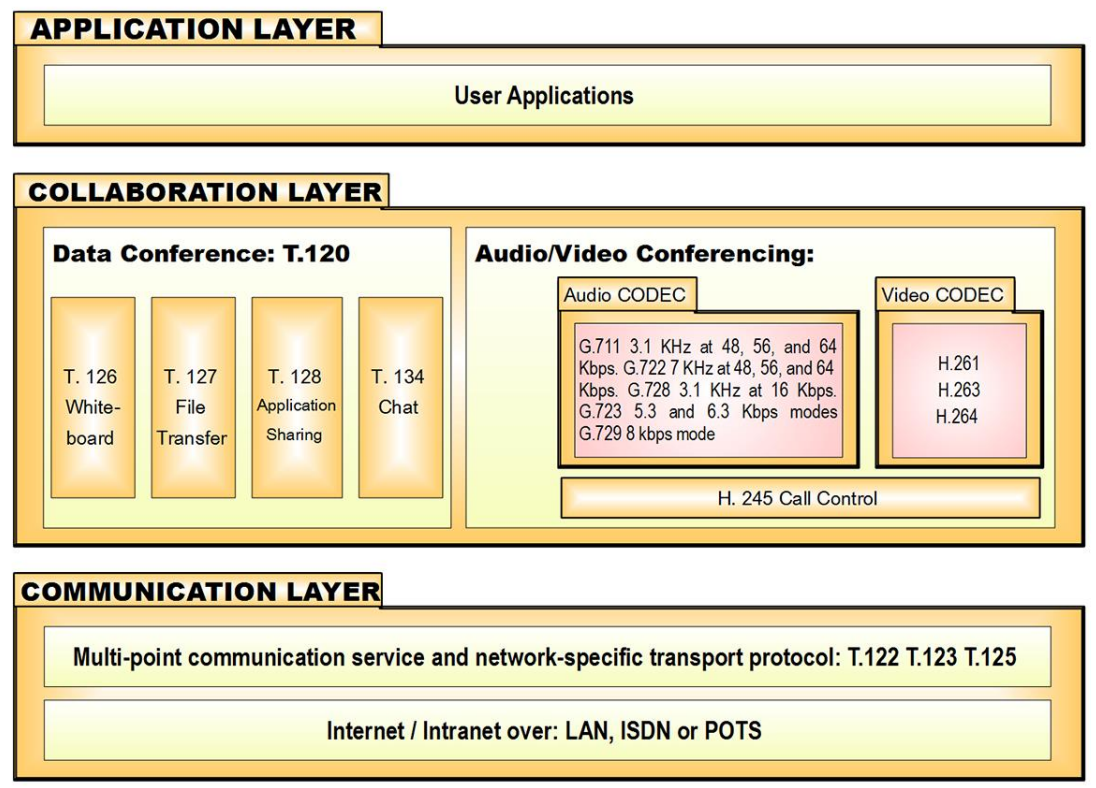

Figure 1. Architecture of communication tools (adapted from (Contero et al., 2002)).

\section{B. Videoconferencing Technology for Collaborative Design}

Research shows that communication mechanisms increase the cooperative awareness of participants in shared environments and play an important role in the resolution of conflicts during the design process (Fu et al., 2013). Collaboration is inherently dynamic and synchronous, i.e. real-time, where communication between two or more individuals takes place simultaneously, like a face-to-face conversation. Although early research suggested that being able to see the conversational partner's faces is surprisingly unimportant in communication, video used to share data does improve communication when it contains the objects being talked about (Karsenty, 1999; Anderson et al., 2000; Fussell et al., 2000). In this context, studies in the area of CSCW show that video conferencing may be the most effective communication tool for distributed teams (Gowan Jr. and Downs, 1994). For example, in a study by (Isaacs and Tang, 1994), researchers found that video improves the ability to show understanding, forecast responses, give non-verbal information, enhance verbal descriptions, manage pauses, and express attitudes (Isaacs and Tang, 1994).

From a user satisfaction point of view, researchers agree on the positive impact of video conferencing for successfully resolving tactical tasks collaboratively (Gowan Jr. and Downs, 1994; Kydd and Ferry, 1994; Olson et al., 1995), but its effectiveness depends on various factors, including the quality and responsiveness of the media (bandwidth), and the specific task that needs to be accomplished (Gajewska et al., 1994; May and Carter, 2001). According to (Isaacs and Tang, 1994), high-quality audio and video, as well as usability, reliability, and support (tools must be simple, easy to use, and as reliable as standard telephone and office applications) are basic requirements for the success of a collaborative videoconferencing system. Appropriate feedback and data access and security are also expected.

Some attempts at communication systems include browser-based services such as Google App Engine, Adobe LiveCycle Collaboration Service, and technology like WebSocket (Hickson, 2009), which provides bidirectional communications channels over TCP. Advanced commercial solutions such as Skype, Apple's FaceTime, or Google Hangouts are also finding its place in the extended enterprise.

Nevertheless, video conferencing sessions cannot be easily managed by current PLM systems, partly because the information cannot be effectively stored and organized in database structures. In addition, the lack of integration between PLM, CAD environments, and communication tools causes a disconnection between the context and the elements of communication (Siltanen and Valli, 2013). In most cases, video conferencing sessions are used in combination with a shared screen, where groups of users interact with a CAD model simultaneously (although only one user can manipulate it at any given time). The result is typically a series of stand-alone tools working independently with no connection to existing CAD software solutions. In this regard, the following drawbacks have been identified (Camba et al., 2014): 
- Additional applications must be run independently from the CAD application to manage the shared environment.

- Integration with existing CAD/PLM systems is difficult, expensive, and often unreliable.

- Editing capabilities in collaborative environments are limited, i.e. when changes need to be made to a model, users are forced to constantly switch between applications.

In addition, when videoconferencing functionality is provided by external commercial tools, other problems appear, including:

- Many video-conferencing tools are not standards-compliant, allowing any vulnerability to pass through corporate firewalls.

- Encryption are typically closed source and prone to man-in-the- middle attacks. Confidentiality and integrity are essential services in any secure video conferencing system.

- Because the service is provided by an external source, outages, downtime, and security cannot be controlled by the organization.

- Numerous video conferencing tools have been reported to use excessive computer resources and network bandwidth.

- Many of the existing tools are untraceable and not auditable, putting organizations that are subject to compliance laws at risk.

For these and other reasons, some system administrators have banned commercial; video conferencing tools on corporate, government, and education networks. In this paper, we propose the addition of collaborative synchronous functionality, particularly real time video/audio communication, to existing CAD systems to facilitate direct point-topoint communication among individuals involved in a particular design activity. Our system is presented a software module that can be integrated in PLM environments using annotated CAD models as mediators for communication. This approach provides a mechanism to interact with other members of the design team without having to use a separate application or leave the CAD environment, and gives corporations full control of their communications technology.

\section{Annotated CAD models}

3D CAD annotations have emerged in recent years as an topic of interest in knowledge management and design engineering research (Yang et al., 2008). This type of annotation uses text and in some cases other forms of content to allow for improved retrieval and exchange of knowledge about the model. In collaborative environments, annotations allow members of a design team to see "who did what" (Hong et al., 1996).

Annotations have facilitated the advancement of the Model-Based Engineering (MBE) paradigm, a modern approach to product development that uses 3D CAD models as the data source for all engineering activities throughout the product life cycle (Lubell et al., 2012). According to (Frechette, 2011), digital models can be understood as vehicles for the delivery of product information, using the 3D views of the model to annotate specific operations, such as manufacturing planning, production simulation, and materials procurement. In this context, modern commercial CAD systems provide "Product and Manufacturing Information" (PMI) modules that support the incorporation of Geometric Dimensioning and Tolerancing (GD\&T), 3D textual annotations, surface finishes, and other product specifications in 3D CAD models. These PMI modules are implementations of standards for digital product definition data practices, such as ASME Y14.41: 2003 or ISO 16792:2006.

Researchers agree that annotations have the ability to capture the rationale behind design decisions (Boujut and Dugdale, 2006; Shum et al., 2006; Li et al., 2009). In our previous work, we developed a broader and more interactive annotation structure that enhances current annotation mechanisms and demonstrated the feasibility of this new model in terms of explicit representation and communication of design intent information (Camba et al., 2014). In this paper, we further enhance this structure by transforming it into a mediator of synchronous communication (video conferencing, shared screen, and instant messaging).

\section{EXTENDED ANNOTATIONS IN PLM ENVIRONMENTS}

The proposed work builds on the previous studies of the authors in the areas of CAD annotation, CAD methodology, and collaborative design (Camba et al., 2014). We define an extended annotation as an annotation structure where information is stored both internally (within the CAD model) and externally (in an external 
repository). The new structure benefits from the existing support for internal annotations available in most CAD systems and the recent formalization of annotation practices by standards such as ASME Y14.41-2003 and ISO 16792:2006.

The system is comprised of three major components: the internal representation, the extended external representation, and a synchronization agent called "annotation manager" that ensures information integrity between the two representations and provides powerful visualization and filtering mechanisms to the user. External representations are enhanced versions the annotations that include relevant data such as creator, date, and links to external documents with the purpose of turning CAD models into more semantic elements and ultimately defining complete annotation-based information systems.

A key attribute of the external representation of the annotations, particularly when a database is used, is the ability to integrate annotation information in a PLM system. Part of the annotation manager resides in the Product Data Management (PDM) module of the PLM and uses the CAD system's application programming interface to extract and insert annotation data. The internal representation of the annotations is stored inside the CAD model and managed directly by the PDM module; external representations are managed as an additional database in the PDM. A representation of the extended annotation model as well as the integration architecture with the PLM system is shown in Figure 2. Other PLM modules have been omitted for clarity.

Integration with CAD applications and PLM environments are fundamental requirements to guarantee the success of 3D mediated communication tools. According to (Siltanen and Valli, 2013), all tools should be based on standard applications used in every company within an extended enterprise and all communication tools should be integrated with the PLM systems so discussions can be linked to PLM objects and discussion history can be retrieved for each object.

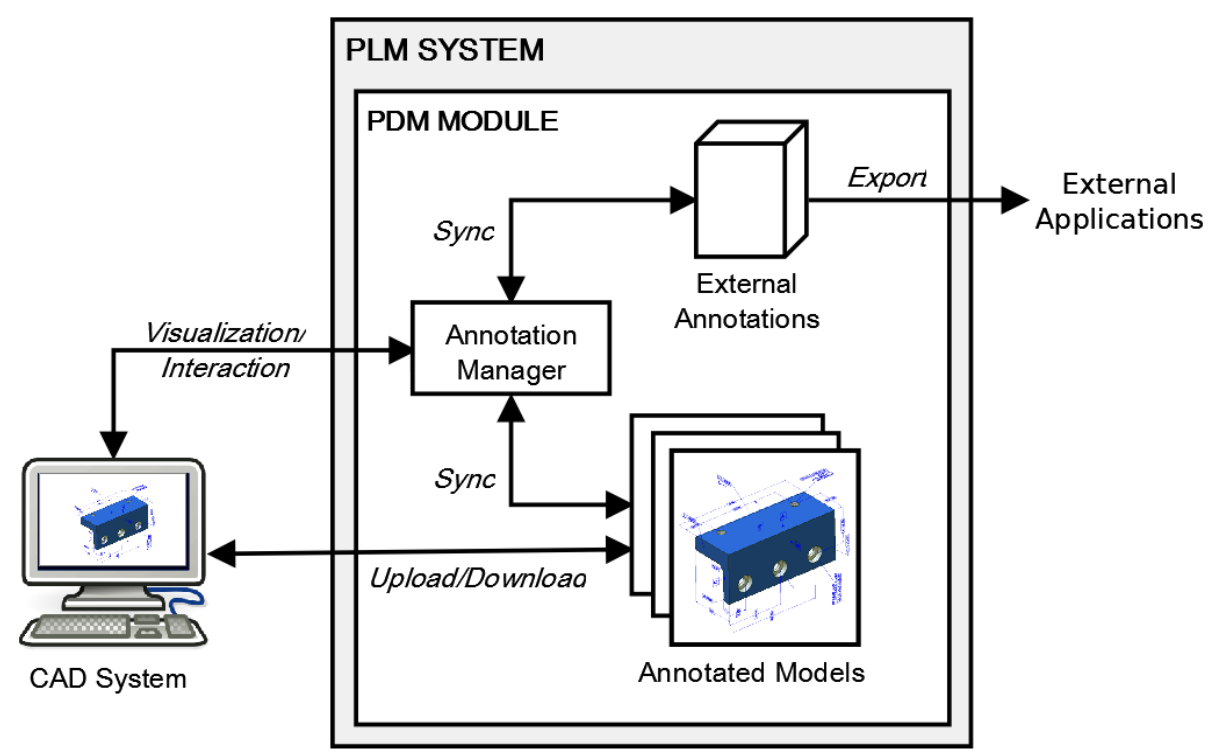

Figure 2. Extended annotations and PLM integration

The availability of annotation information in PLM repositories enables a more efficient use of shared CAD models by facilitating access to design information. For example, annotation information can be used by external applications to analyze model quality, design practices, or study interactions between users in collaborative environments.

In addition, automatic mechanisms to save and manage historical information about particular decisions and design changes can be implemented. This information can be used to study the evolution of CAD models and design intent information in collaborative environments, or analyze team interactions over time. Annotation records also allow users to restore previous annotations or revert back to earlier annotation states of the model, if necessary. 


\section{SYNCHRONOUS COMMUNICATION FUNCTIONALITY}

In a scenario where heavily annotated CAD models are used and shared by various members of a design team to communicate design intent and/or manufacturing information, specific questions regarding annotation information may need to be answered and certain aspects of the model may need to be explained by designers when annotations are not clear. An efficient mechanism to communicate with users that are involved in a particular design task and create specific annotations is desirable.

We propose the addition of synchronous communication functionality to the annotation system developed in our previous work (described above). The new capabilities are presented as an extension to our annotation manager tool, which was originally developed to improve user interaction with heavily annotated models by combining internal and external representations of the annotations. In our approach, 3D annotations are used as information-rich mediators between participants where user information is available per annotation. Users interacting with a shared model can utilize annotations as communication links to contact other users. Users include model creators, consumers of CAD models created by other participants, and annotators.

The proposed solution is presented as a complimentary module that provides instant messaging, IP-based point-topoint video communication, and screen sharing services to the CAD application. Communication is managed through the PLM system but, once established, it does not rely on a central server. The architecture of the system is shown in Figure 3. Traffic between the CAD system and the PDM module (to synchronize CAD files, update versions, etc) is shown with dashed lines. Data flow to and from the annotation manager and the communications module is indicated with solid lines.

For any CAD model in a design project, participants' information is linked to the annotations. User information is stored per annotation as part of the external annotation database in the PLM system. This information is connected to a new table that contains the contact information (IP address and port) required by the communication module to establish a session. If an annotation is selected in the CAD model, the annotator's information is available to the user and a video call or screen sharing session can be requested.

When a communication session is started, the annotation manager retrieves user information from the corresponding table in the PLM system and creates a point-to-point connection between the two users. Commands are available to establish instant messaging, video calls, and shared screen sessions with the selected participants.

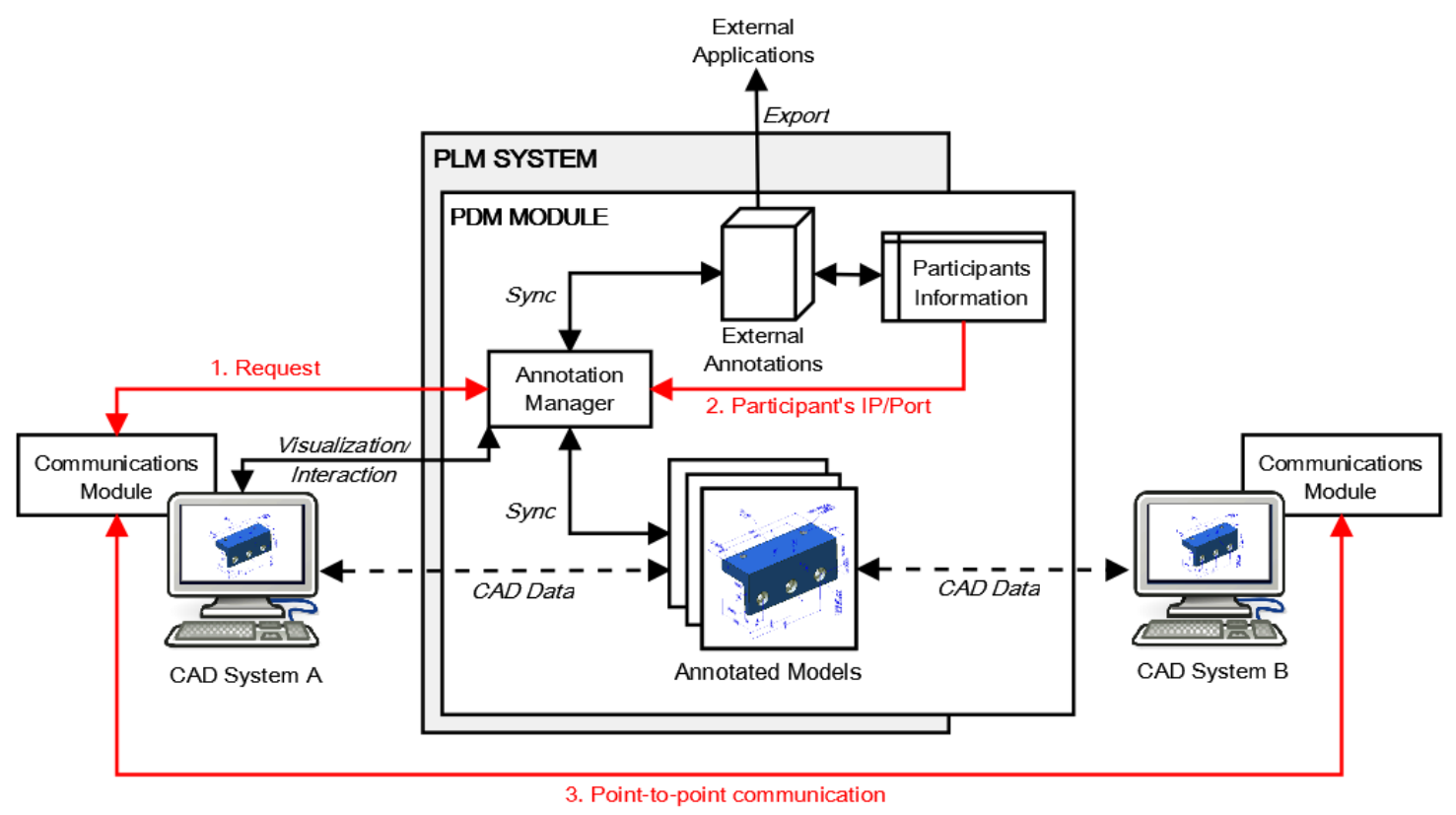

Figure 3. System architecture

In our previous work (Camba et al., 2014), we created a software plug-in that connects with the CAD environment (DS SolidWorks ${ }^{\circledR}$, in our case) as an extra set of tools by using the Application Programming Interface (API) capabilities provided by the CAD application. To evaluate the new communications module, we improved our 
original prototype by adding a point-to-point video conferencing service. The software was designed with flexibility in mind so it could be easily ported to other platforms and packages.

The communications module was developed as a multithreaded application (see Figure 4). To ensure responsiveness to other users' requests, the module implements a server thread that listens to incoming connections. A separate client thread is used to request communication and initiate the session. Additional responsibilities of the client thread include receiving, decoding, and displaying the video frames.

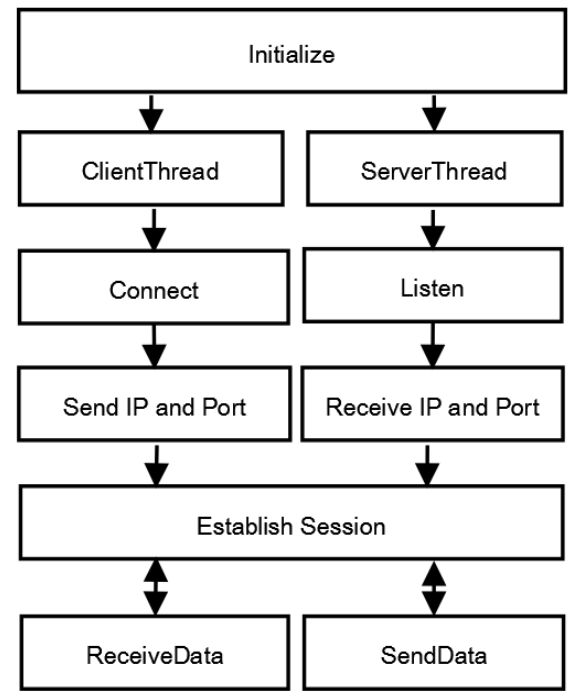

Figure 4. Multithreaded architecture

The User Datagram Protocol (UDP) was used for video transmission to guarantee a continuous data stream. Because of the time-sensitive nature of the data (real-time audio and video), dropping packets is preferable to waiting for delayed packets. Therefore, the lack of retransmission delays makes UDP suitable for real-time streaming applications. For initial testing and demonstration purposes, our current prototype transmits both audio and video in an uncompressed form. Because video conferencing applications require real-time compression in which frames are quickly captured and sent to the network, future plans include the incorporation of high-definition video compression techniques such as H.264.

The workflow using the annotation manager with the video conferencing module is illustrated in Figure 5. First, the user loads an annotated model from the PLM system onto her workstation and selects the annotation of interest directly from the 3D model or via the annotation manager interface, where all model annotations are listed in tabular form showing all related metadata (type, creator, date, etc). When an annotation is selected, it is highlighted both in the 3D model and in the annotation manager area, which provides an effective visual cue to the user. Next, a communication session can be started by selecting a command from the list that is available per annotation. By clicking "contact annotator," the annotation manager automatically connects to the PLM system and requests the contact information (IP address and port) of the annotator. The PLM system sends the information back to the client and the video call is started. At the same time, the user on the other end receives a notification and is prompted to accept the request. When the session is established, all data transmissions between the participants take place without PLM intervention. 


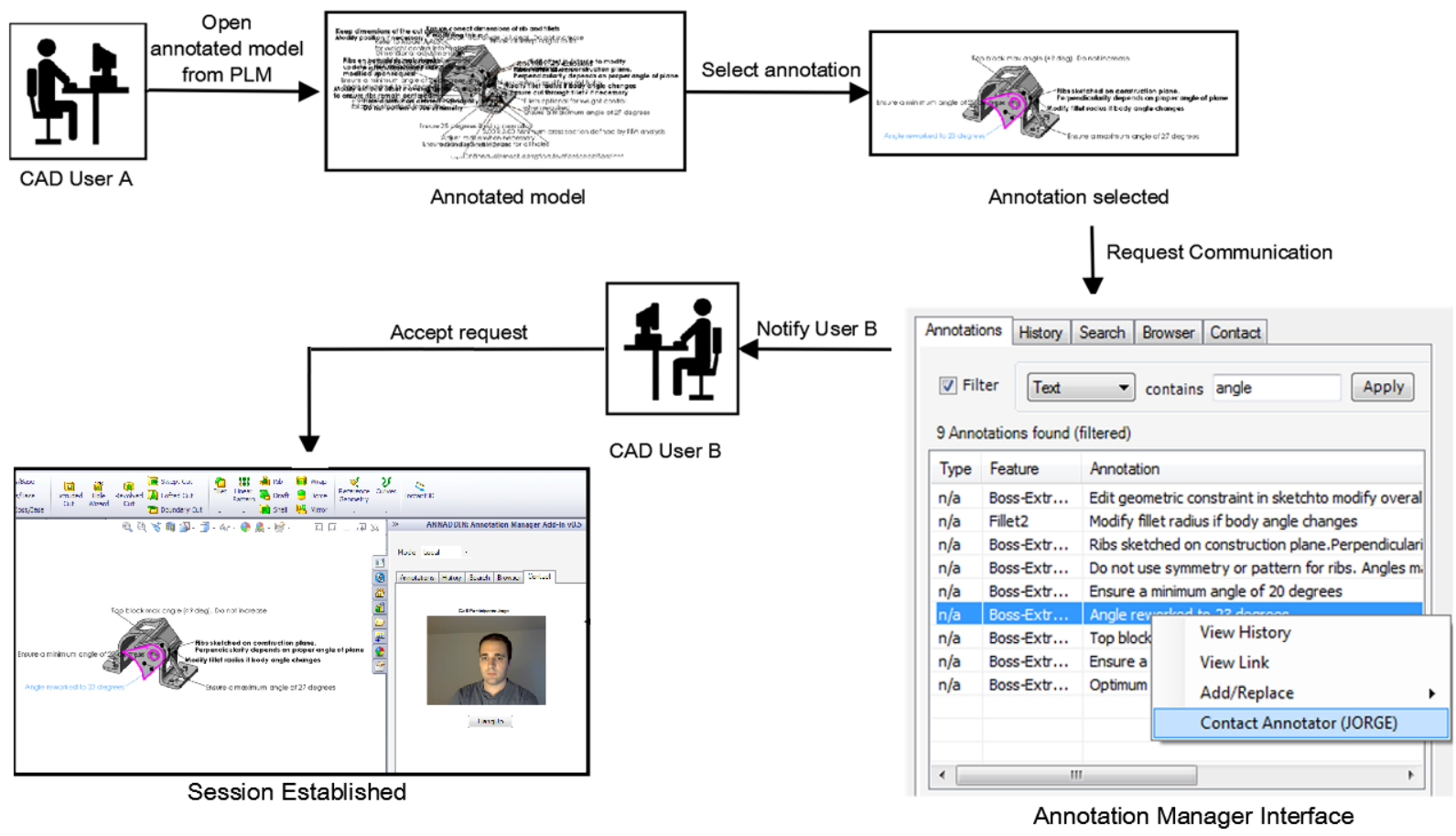

Figure 5. Workflow

\section{CONCLUSIONS AND FUtURE WORK}

Geographically dispersed teams rely on information and communication technologies (ICTs) to communicate and collaborate. Our research is focused on addressing some of the intrinsic limitations of traditional model-based engineering systems and the current representation of design information. The use of model annotations as communication elements have the potential to facilitate collaborative development and lead to significant gains in terms of quality and design reusability.

Our extended annotation mechanism was significantly enhanced by incorporating synchronous communication functionality shows that 3D CAD models can be utilized to a much larger extent in collaborate design environments throughout the product life cycle than they currently are. The inherent constraints of using external applications that are disconnected from design environments cause integration and compatibility problems with existing systems. In addition, users must learn yet another program and be constantly switching between multiple programs when used in real design scenarios. For these and other reasons, it is difficult for these applications to find acceptance in the concurrent engineering workflow.

In this paper, we have proposed a novel architecture to collaborative engineering based on annotated models that incorporates synchronous communication functionality "per annotation." In our system, users can select a specific annotation from the model and establish a communication session with other users involved with the annotation directly from the CAD environment.

Despite the fact that the communications module was shown to a number of engineers and product designers in an informal setting and positive initial reactions were observed, a formal validation with users in production environments and a comparative study with other communication tools are still necessary. Also, performance testing and analysis of the impact in the modeling workflow is required for situations where multiple users are connected to the same conference session.

Future efforts will be dedicated to improve streaming and video quality by using different video compression systems, such as H.264, and the use of advanced buffering techniques to order incoming packets and ensure a constant flow of frames. Flow control algorithms will be necessary to prevent buffer underflows (which may result in gaps during video display) and overflows (which result in frame loss). 
Finally, video recording capabilities and automatic captioning can be implemented for subsequent processing and playback of the video stream. This would allow users to export the information discussed in the video call to textual formats, so it can be managed and processed by other users and applications. The availability of the information in textual form can make this otherwise inaccessible information a data source for automatic data mining and knowledge extraction techniques.

\section{REFERENCES}

[1] Abrahamson S, Wallace D, Senin N, Sferro P (2000) Integrated design in a service marketplace. ComputerAided Design 32(2):97-107.

[2] Ahmed S (2005) Encouraging reuse of design knowledge: A method to index knowledge. Design Studies 26:565-592.

[3] Alavi M, Tiwana A (2002) Knowledge integration in virtual teams: The potential role of KMS. Journal of the American Society for Information Science and Technology 53:1029-1037.

[4] Ameri F, Dutta D (2005) Product lifecycle management: closing the knowledge loops. Computer-Aided Design and Applications 2(5):577-590.

[5] Anderson AH, Smallwood L, MacDonald R, Mullin J, Fleming A, O'Malley C (2000) Video data and video links in mediated communication: what do users value? International Journal of Human-Computer Studies 52(1):165-187.

[6] Arias E, Eden H, Fischer G, Gorman A, Scharff E (2000) Transcending the individual human mind-creating shared understanding through collaborative design. ACM Transactions on Computer-Human Interaction (TOCHI) 7(1): 84-113.

[7] Bai J, Gao S, Tang W, Liu Y, Guo S (2010) Design reuse oriented partial retrieval of CAD models. ComputerAided Design 42:1069-1084.

[8] Barley WC, Leonardi PM, Bailey DE (2012) Engineering Objects for Collaboration: Strategies of Ambiguity and Clarity at Knowledge Boundaries. Human Communication Research 38:280-308.

[9] Boujut JF, Dugdale J (2006) Design of a 3D Annotation Tool for Supporting Evaluation Activities in Engineering Design. Cooperative Systems Design, COOP 6:1-8.

[10] Camba J, Contero M, Johnson M, Company P (2014) Extended 3D annotations as a new mechanism to explicitly communicate geometric design intent and increase CAD model reusability. Computer-Aided Design 57:61-73.

[11] Camba J, Contero M, Salvador-Herranz G (2014) Speak with the annotator: promoting interaction in a knowledge-based CAD environment built on the extended annotation concept. Proceedings of the 2014 IEEE 18th International Conference on Computer Supported Cooperative Work in Design (CSCWD), 196-201.

[12] Chudoba KM, Wynn E, Lu M, Watson-Manheim MB (2005) How virtual are we? Measuring virtuality and understanding its impact in a global organization. Information Systems Journal 15(4):279-306.

[13] Contero M., Vila C, Aleixos N (2002) Product data quality and collaborative engineering. IEEE Computer Graphics and Applications 22(3):32-42.

[14] Cutkosky MR, Tenenbaum JM, Glicksman J (1996) Madefast: collaborative engineering over the Internet. Communications of the ACM 39(9):78-87.

[15] Danesi F, Gardan N, Gardan Y (2006) Collaborative Design: From Concept to Application. Geometric Modeling and Imaging--New Trends 90-96.

[16] Durstewitz M, Kiefner B, Kueke R, Putkonen H, Repo P, Tuikka T (2002) Virtual collaboration environment for aircraft design. Proceedings of the IEEE 6th International Conference on Information Visualisation 502-507.

[17] Fisher D, Brush AJ, Gleave E, Smith MA (2006) Revisiting Whittaker and Sidner's “email overload” ten years later. Proceedings of the 2006 20th anniversary conference on Computer supported cooperative work. ACM, Banff 
[18] Fonseca MJ, Henriques E, Silva N, Cardoso T, Jorge JA (2006) A collaborative CAD conference tool to support mobile engineering. Rapid Product Development (RPD’06), Marinha Grande, Portugal.

[19] Frechette SP (2011) Model Based Enterprise for Manufacturing. Proceedings of the 44th CIRP International Conference on Manufacturing Systems.

[20] Fu WX, Bian J, Xu YM (2013) A video conferencing system for collaborative engineering design. Applied Mechanics and Materials 344:246-252.

[21] Fuh JYH, Li WD (2005) Advances in collaborative CAD: The-state-of-the art. Computer-Aided Design 37:571581.

[22] Fussell SR, Kraut RE, Siegel J (2000) Coordination of communication: Effects of shared visual context on collaborative work. Proceedings of the 2000 ACM conference on Computer supported cooperative work, 21-30.

[23] Gajewska H, Kistler J, Manasse MS, Redell D (1994) Argo: a system for distributed collaboration. Proceedings of the ACM Second International Conference on Multimedia, San Francisco, CA, USA, 433-440.

[24] Gantz J, Reinsel D, Chute C, Schlichting W, Mcarthur J, Minton S, Xheneti I, Toncheva A, Manfrediz A (2007) The expanding digital universe: a forecast of worldwide information growth Through 2010. IDC, Massachusetts.

[25] Gowan Jr JA, Downs JM (1994) Video conferencing human-machine interface: a field study. Information and Management 27(6):341-356.

[26] Gupta A, Mattarelli E, Seshasai S, Broschak J (2009) Use of collaborative technologies and knowledge sharing in co-located and distributed teams: Towards the 24-h knowledge factory. The Journal of Strategic Information Systems 18:147-161.

[27] Hickson I (2009) The Web Socket protocol IETF, Standards Track

[28] Hong J, Toye G, Leifer LJ (1996) Engineering design notebook for sharing and reuse. Computers in Industry 29:27-35.

[29] Isaacs EA, Tang JC (1994) What video can and cannot do for collaboration: a case study. Multimedia Systems 2(2):63-73.

[30] Karsenty L (1999) Cooperative work and shared visual context: An empirical study of comprehension problems in side-by-side and remote help dialogues. Human-Computer Interaction 14(3):283-315.

[31] Kydd CT, Ferry DL (1994) Managerial use of video conferencing. Information and Management 27(6):369375.

[32] Lahti H, Seitamaa-Hakkarainen P, Hakkarainen K (2004) Collaboration patterns in computer supported collaborative designing. Design Studies 25:351-371.

[33] Leenders RTA, Van Engelen JM, Kratzer J (2003) Virtuality, communication, and new product team creativity: a social network perspective. Journal of Engineering and Technology Management 20(1):69-92.

[34] Levitt RE, Jin Y, Dym CL (1991). Knowledge-based support for management of concurrent, multidisciplinary design. Artificial Intelligence for Engineering, Design, Analysis and Manufacturing 5(2):77-95.

[35]Li C, McMahon C, Newnes L (2009) Annotation in Product Lifecycle Management: A Review of Approaches. Proceedings of the ASME International Design Engineering Technical Conferences and Computers and Information in Engineering Conference, DETC2009. Vol. 2. New York: ASME, 797-806.

[36] Li WD, Lu WF, Fuh JY, Wong YS (2005) Collaborative computer-aided design-research and development status. Computer-Aided Design 37(9):931-940.

[37]Londono F, Cleetus KJ, Nichols DM, Iyer S, Karandikar HM, Reddy SM, Potnis SM, Massey B, Reddy A, Ganti V (1992) Coordinating a virtual team. CERC-TR-RN-92-005, Concurrent Engineering Research Centre, West Virginia University, West Virginia.

[38]Lubell J, Chen K, Horst J, Frechette S, Huang P (2012) Model Based Enterprise/Technical Data Package Summit Report. NIST Technical Note 1753. 
[39] May A, Carter C (2001) A case study of virtual team working in the European automotive industry. International Journal of Industrial Ergonomics 27(3):171-186.

[40] Olson JS, Olson GM, Meader DK (1995) What mix of video and audio is useful for small groups doing remote real-time design work? Proceedings of the SIGCHI conference on Human factors in computing systems. ACM Press/Addison-Wesley Publishing Co.

[41] Ping-Hung H, Mishra CS, Gobeli DH (2003) The return on R\&D versus capital expenditures in pharmaceutical and chemical industries. IEEE Transactions on Engineering Management 50:141-150.

[42] Sharma A. (2005). Collaborative product innovation: integrating elements of CPI via PLM framework. Computer-Aided Design 37(13):1425-1434.

[43] Shum SJB, Selvin AM, Sierhuis M, Conklin J, Haley CB, Nuseibeh B (2006) Hypermedia Support for Argumentation-Based Rationale: 15 Years on from Gibis and Qoc. Rationale Management in Software Engineering, 111-132.

[44] Siltanen P, Valli S (2013). Web-based 3D Mediated Communication in Manufacturing Industry. Concurrent Engineering Approaches for Sustainable Product Development in a Multi-Disciplinary Environment, 11811192. Springer London.

[45] Stark J (2011). Product lifecycle management. 1-16. Springer London.

[46] Tavčar J, Potočnik U, Duhovnik J (2013) PLM Used as a Backbone for Concurrent Engineering in Supply Chain. Concurrent Engineering Approaches for Sustainable Product Development in a Multi-Disciplinary Environment, 681-692. Springer London.

[47] Tay FEH, Ming C (2001) A shared multi-media design environment for concurrent engineering over the internet. Concurrent Engineering 9(1):55-63.

[48] Tay FEH, Roy A (2003) CyberCAD: a collaborative approach in 3D-CAD technology in a multimediasupported environment. Computers in Industry 52(2):127-145.

[49] Toussaint J, Cheng K (2002) Design agility and manufacturing responsiveness on the web. Integrated Manufacturing Systems 13(5):328-339.

[50] Tsoi KN, Rahman SM (1996) Media-on-demand multimedia electronic mail: a tool for collaboration on the web. Proceedings of the 5th IEEE International Symposium on High Performance Distributed Computing. IEEE Computer Society.

[51] Upton DM, Mcafee A (1999) The real virtual factory. Harvard Business School Press, 69-89.

[52] Vila C, Estruch A, Siller HR, Abellán JV, Romero F (2007). Workflow methodology for collaborative design and manufacturing. Cooperative Design, Visualization, and Engineering 42-49, Springer Berlin Heidelberg.

[53] Wasiak J, Hicks B, Newnes L, Dong A, Burrow L (2010) Understanding engineering email: the development of a taxonomy for identifying and classifying engineering work. Research in Engineering Design 21(1):43-64.

[54] Wasko MM, Faraj S (2005) Why should I share? Examining social capital and knowledge contribution in electronic networks of practice. MIS Quarterly: Management Information Systems 29:35-57.

[55] Yang QZ, Zhang Y, Miao CY, Shen ZQ (2008) Semantic Annotation of Digital Engineering Resources for Multidisciplinary Design Collaboration. ASME 2008 International Design Engineering Technical Conferences and Computers and Information in Engineering Conference, 617-624. American Society of Mechanical Engineers.

[56] You CF, Chao SN (2006) Multilayer architecture in collaborative environment. Concurrent Engineering Research and Applications 14(4):273-281.

[57] Yuan YC, Fulk J, Monge PR, Contractor N (2010) Expertise directory development, shared task interdependence, and strength of communication network ties as multilevel predictors of expertise exchange in transactive memory work groups. Communication Research 37: 20-47. 